\title{
Type d'homotopie des treillis et treillis des sous-groupes d'un groupe fini
}

\author{
Charles Kratzer et Jacques Thévenaz
}

A tout ensemble (partiellement) ordonné $L$, on peut associer un complexe simplicial $|L|$ dont les $n$-simplexes sont les chaînes $a_{0}<a_{1}<\cdots<a_{n}$ dans $L$. Si $\bar{L}$ est un treillis fini avec élément minimal 0 et élément maximal 1 , on s'intéresse aux propriétés homotopiques de complexe $|L|$ où $L=\bar{L}-\{0,1\}$. Notons qu'il est nécessaire d'enlever 0 et 1 sinon le complexe est un cône, donc contractile. Soit $a \in L$ un point fixé; rappelons qu'un complément de a est un élément $c \in L$ tel que $c \wedge a=0$ et $c \vee a=1$. On démontre tout d'abord (Proposition 1.8) que s'il existe un point a sans complément, alors $|L|$ est contractile. Certains points très particuliers de $L$, appelés pivots, permettent de décrire le type d'homotopie de $|L|$. Le théorème principal (Théorème 2.4) affirme en effet qu'on peut entièrement connaître $|L|$ à homotopie près en fonction de sous-complexes déterminés par un pivot et ses compléments. On montre alors (Proposition 3.1) que tout point maximal d'un treillis modulaire est un pivot, et il en résulte (Théorème 3.4) que le complexe associé à un treillis modulaire a le type d'homotopie d'un bouquet de sphères de dimension $n-2$, où $n$ est la longueur du treillis. La version homologique de ce résultat est dûe à Folkman [F] pour les treillis géométriques. En particulier, on calcule aisément le type d'homotopie du treillis des sous-espaces d'un espace vectoriel fini et on retrouve ainsi la version homologique de ce résultat, dûe à Lusztig [L].

Si maintenant $\bar{L}$ est le treillis des sous-groupes d'un groupe fini $G$, on étudie d'abord quelques conditions pour que $|L|$ soit contractile. On montre que si $N$ est un sous-groupe de $G$ normal abélien et semi-simple comme $\mathbb{Z} G$-module ( $G$ agissant par conjugaison sur $N$ ), alors $N$ est un pivot (Théorème 4.7). Il en résulte que si $G$ est résoluble, le complexe associé a le type d'homotopie d'un bouquet de sphères de dimension $n-2$, où $n$ est la longueur d'une suite principale de $G$ (Corollaire 4.10). Le nombre de ces sphères (qui n'est autre que la caractéristique d'Euler réduite du complexe) est facilement calculable en fonction du nombre de compléments de chaque sous-groupe normal d'une suite principale. On retrouve là un résultat de notre article $[\mathrm{K}-\mathrm{T}]$ où seule la fonction de Möbius du treillis était étudiée. En effet, il est bien connu [R] que cette fonction coīncide avec la caractéristique d'Euler réduite du complexe associé. 
Le premier paragraphe est consacré aux préliminaires et à quelques résultats généraux. Le théorème principal est démontré au paragraphe 2. Les applications du théorème aux treillis modulaires font l'objet du troisième paragraphe. Enfin, dans le dernier paragraphe, nous étudions le complexe associé au treillis des sous-groupes d'un groupe fini.

\section{Préliminaires}

Soit $E$ un ensemble (partiellement) ordonné et soit $|E|$ le complexe simplicial associé: un $n$-simplexe de $|E|$ est une chaîne $a_{0}<a_{1}<\cdots<a_{n}$ dans $E$. Une application croissante $f: E \rightarrow F$ entre ensembles ordonnés induit une application simpliciale $|f|:|E| \rightarrow|F|$. Cette construction permet d'appliquer aux ensembles ordonnés des concepts topologiques. Ainsi on dira par exemple que $E$ est contractile si $|E|$ l'est et que $f$ est une équivalence d'homotopie si $|f|$ l'est.

Nous allons faire un large usage des résultats suivants $[Q, \S 1]$ :

1.1. Si $E$ possède un plus grand (ou plus petit) élément $a$, alors $|E|$ est un cône de sommet a. En particulier, E est contractile.

1.2. Si $f, g: E \rightarrow F$ sont deux applications croissantes telles que $f(x) \geqq g(x)$ pour tout $x \in E$, alors $|f|$ est homotope à $|g|$.

Ces deux propriétés impliquent la suivante:

1.3. Soit $f: E \rightarrow E$ une application croissante et $a \in f(E)$. Si $x \leqq f(x) \geqq a$ pour tout $x \in E$, alors $|E|$ a le type d'homotopie d'un cône de sommet $a$. En particulier, $E$ est contractile.

On a le même résultat en renversant les inégalités.

1.4. THÉORÈME A (Quillen). Soit $f: E \rightarrow F$ une application croissante. Pour $y \in F$, on considère la "fibre" $f / y=\{x \in E ; f(x) \leqq y\}$. Si f/y est contractile pour tout $y \in F$, alors $f$ est une équivalence d'homotopie.

On a le même résultat en remplaçant dans l'énoncé $f / y$ par $y \backslash f=\{x \in E ; y \leqq f(x)\}$.

Remarque 1.5. On utilisera ce théorème dans le cas d'une inclusion $i: E \hookrightarrow F$. Pour $y \in E,|i / y|$ est un cône de sommet $y$, si bien qu'il suffit, pour être dans les hypothèses du théorème, de montrer que $i / y$ est contractile si $y \in F-E$. 
Soit $E$ un ensemble ordonné et $a, b \in E$. On définit les intervalles $[a, b]=$ $\{x \in E ; a \leqq x \leqq b\}$ et $(a, b)=\{x \in E ; a<x<b\}$. Si $\vec{E}$ est un ensemble ordonné possédant un unique élément minimal 0 et un unique élément maximal 1 (si bien que $\vec{E}=[0,1])$, on s'intéressera à $E=(0,1)$. Cela s'applique en particulier aux treillis finis, car ils ont nécessairement un unique élément minimal et un unique élément maximal.

PROPOSITION 1.6. Soit $\vec{E}=[0,1]$ un ensemble ordonné fini et $E=(0,1)$. Soit $A$ un sous-ensemble de $E$ tel que $(0, a)$ est contractile pour tout $a \in A$. Alors l'inclusion $E-A \leftrightarrow E$ est une équivalence d'homotopie.

Preuve. Soit $M$ l'ensemble des éléments maximaux de $A$. Il suffit de montrer que $i: E-M \hookrightarrow E$ est une équivalence d'homotopie, puis de répéter l'opération. Par le Théorème $A$ et la Remarque 1.5 , il suffit de montrer que si $y \in M, i / y$ est contractile. Mais comme deux éléments de $M$ ne sont pas comparables, tout $x<y$ appartient $\grave{a} E-M$, si bien que $i / y=(0, y)$. Le résultat s'ensuit car $(0, y)$ est contractile par hypothèse.

Remarque. Dualement, $E$ garde le même type d'homotopie si on lui enlève des points a pour lesquels $(a, 1)$ est contractile.

Dans la suite, $\bar{L}$ désignera un treillis fini, c'est-à-dire un ensemble fini (partiellement) ordonné $\vec{L}$ tel que toute paire d'éléments $a, b$ possède un supremum, noté $a \vee b$ et un infimum, noté $a \wedge b$; nous noterons 0 son unique élément minimal, 1 son unique élément maximal et $L=(0,1)$. Rappelons que si $a \in \bar{L}$, un complément de a dans $\bar{L}$ est un élément $c \in \bar{L}$ tel que $c \wedge a=0$ et $c \vee a=1$.

LEMME 1.7. Soit $a, x \in \bar{L}$ tels que $a \vee x=1$. Si $c$ est un complément de $a \wedge x$ dans $[0, x]$, alors $c$ est un complément de a dans $[0,1]=\bar{L}$.

Preuve. On a $c \vee a \geqq c \vee(a \wedge x)=x$, si bien que $c \vee a \geqq x \vee a=1$ et donc $c \vee a=$ 1. Par ailleurs, $c \wedge a=c \wedge x \wedge a=0$.

Dualement, si $a \wedge x=0$ et si $c$ est un complément de $a \vee x$ dans $[x, 1]$, alors $c$ est un complément de a dans $\tilde{L}$.

PROPOSITION 1.8. Soit $\bar{L}=[0,1]$ un treillis fini et $L=(0,1)$. S'il existe $a \in L$ ne possédant pas de complément dans $\bar{L}$, alors $L$ est contractile. 
Preuve. Soit $X=\{x \in L ; a \vee x=1\}$. Par le lemme 1.7, si $x \in X$, alors $a \wedge x$ n'a pas de complément dans $[0, x]$. Par induction sur le cardinal de $L$, on peut supposer que $(0, x)$ est contractile. Remarquons qu'au départ de l'induction, $X$ est nécessairement vide. Par la Proposition 1.6, $L-X \hookrightarrow L$ est alors une équivalence d'homotopie et il suffit donc de montrer que $L-X$ est contractile. Or pour tout $y \in L-X$, on a $y \vee a \in L-X$ et $y \leqq y \vee a \geqq a$. Ainsi par 1.3, $L-X$ est contractile.

Remarque. La réciproque de la Proposition 1.8 n'est pas vraie. Soit en effet $L=(0,1)=\{a, b, c, d, e, f\}$ avec $a>b<c>d<e>f$. Alors $L$ est contractile et tout point possède un complément dans $\vec{L}$. Par ailleurs, Björner démontre dans $\left[\mathrm{B}_{2}\right]$ par d'autres méthodes un résultat plus général: pour qu'un treillis soit non-contractile, il faut qu'il soit fortement complémenté.

\section{Pivots et type d'homotopie}

Soit $\bar{L}=[0,1]$ un treillis fini, $L=(0,1)$ et $a \in L$. L'ensemble des compléments de a dans $\vec{L}$ est noté $a^{\perp}$. On suppose que $a^{\perp}$ est non vide sinon $L$ est contractile

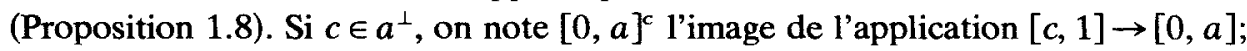
$x \mapsto x \wedge a$.

LEMME 2.1. $[0, a]^{c}=\{b \in[0, a] ;(b \vee c) \wedge a=b\}$

Preuve. Si $b \in[0, a]^{c}$, il existe $x \in[c, 1]$ tel que $x \wedge a=b$. Comme $b \leqq x$ et $c \leqq x$, $b \vee c \leqq x$ et donc $(b \vee c) \wedge a \leqq x \wedge a=b$. Par ailleurs, clairement $b \leqq(b \vee c) \wedge a$ si bien que $(b \vee c) \wedge a=b$. Réciproquement, si $b=(b \vee c) \wedge a$, alors visiblement $b \in$ $[0, a]^{c}$.

On note aussi $(0, a)^{c}=[0, a]^{c}-\{0, a\}$. Remarquons que si $c \in a^{\perp}$, alors $a \in c^{\perp}$ et donc $[0, c]^{a}$ a un sens. Pour $c \in a^{\perp}$, on définit encore les sous-ensembles suivants:

$$
\begin{aligned}
& \bar{L}_{c}=\left\{x \in \bar{L} ; x=x_{a} \vee x_{c}, x_{a} \in[0, a]^{c}, x_{c} \in[0, c]^{a}\right\} . \\
& \bar{L}_{c}^{\prime}=\left\{x \in \bar{L} ; x=x_{a} \vee x_{c}, x_{a} \in[0, a]^{c}, x_{c} \in[0, c]\right\} . \\
& L_{c}=\bar{L}_{c} \cap L=\bar{L}_{c}-\{0,1\} . \\
& L_{c}^{\prime}=\bar{L}_{c}^{\prime} \cap L=\bar{L}_{c}^{\prime}-\{0,1\} .
\end{aligned}
$$

Rappelons que si $X$ et $Y$ sont deux ensembles ordonnés, alors $X \times Y$ l'est aussi 
pour l'ordre suivant:

$$
(x, y) \leqq\left(x^{\prime}, y^{\prime}\right) \text { si } x \leqq x^{\prime} \quad \text { et } \quad y \leqq y^{\prime}
$$

LEMME 2.2. a) Si $x=x_{a} \vee x_{c} \in \bar{L}_{c}^{\prime}$, alors $x_{a}=x \wedge a$.

b) Si $x=x_{a} \vee x_{c} \in \bar{L}_{c}$, alors $x_{a}=x \wedge a$ et $x_{c}=x \wedge c$.

c) $\bar{L}_{c}$ est isomorphe au produit $[0, a]^{c} \times[0, c]^{a}$.

Preuve. a) Clairement, $x_{a} \leqq x \wedge a$ et il s'agit de montrer l'autre inégalité. Comme $x_{a} \in[0, a]^{c}, x_{a}=z \wedge a$ où $z=x_{a} \vee c$ (Lemme 2.1). Alors $x=x_{a} \vee x_{c} \leqq$ $x_{a} \vee c=z$ et donc $x \wedge a \leqq z \wedge a=x_{a}$.

b) Résulte de a) et de l'échange des rôles de $a$ et $c$.

c) L'application $[0, a]^{c} \times[0, c]^{a} \rightarrow \bar{L}_{c} ;\left(x_{a}, x_{c}\right) \mapsto x_{a} \vee x_{c}$ est croissante et surjective par définition de $\bar{L}_{c}$. Elle est injective car par b), l'écriture $x=x_{a} \vee x_{c}$ est unique.

Remarque 2.3. Si $x=x_{a} \vee x_{c} \in \check{L}_{c}^{\prime}$, alors $x_{c}$ n'est pas unique. Le plus grand tel $x_{c}$ est égal à $x \wedge c$.

Notons encore $L^{\prime}=\bigcup_{c \in a^{\lrcorner}} L_{c}^{\prime}$. On dira que $a \in L$ est un pivot si les trois conditions suivantes sont satisfaites:

(i) $[0, a]^{c}$ ne dépend pas de $c \in a^{\perp}$.

(ii) Deux compléments distincts de a ne sont pas comparables: si $c, d \in a^{\perp}$ avec $c \leqq d$, alors $c=d$.

(iii) Si $x_{1}, \ldots, x_{n} \in L^{\prime}$ avec $x_{1}<x_{2}<\cdots<x_{n}$, alors il existe $c \in a^{\perp}$ tel que $x_{i} \in L_{c}^{\prime}$ pour tout $1 \leqq i \leqq n$.

En particulier, si $a^{\perp}=\varnothing$, alors a est un pivot. Avant d'énoncer le théorème principal, rappelons quelques concepts topologiques:

Cône: Si $X$ est un espace topologique, $X \times[0,1] / X \times\{1\}$ est le cône sur $X$, noté $C X$.

Suspension: Si $X$ est un espace topologique, $X \times[0,1] / X \times\{0\} \cup X \times\{1\}$ est la suspension de $X$, notée $\sum X$. Par conséquent, si $Y=Y_{1} \cup Y_{2}$ est tel que $X=$ $Y_{1} \cap Y_{2}$ et si chaque $Y_{i}$ est un cône sur $X$, alors $Y$ est la suspension de $X$. En particulier, si $S^{n}$ désigne la sphère de dimension $n, \sum S^{n}=S^{n+1}$.

Joint: $\mathrm{Si} X_{1}$ et $X_{2}$ sont deux espaces topologiques, $Y_{1}=C X_{1} \times X_{2}, Y_{2}=$ $X_{1} \times C X_{2}$, alors $Y=Y_{1} \cup_{x_{1} \times X_{2}} Y_{2}$ est le joint de $X_{1}$ et $X_{2}$, noté $X_{1} * X_{2}$. En particulier, $S^{n} * S^{m}=S^{n+m+1}$. Il est commode de poser $S^{-1}=\varnothing$ et de définir le cône sur $\varnothing$ comme étant un point. Alors $\sum S^{-1}=S^{0}$ et la formule du joint des sphères s'étend à $n, m \geqq-1$. Finalement, $V_{i \in I} X_{i}$ désigne le bouquet des espaces (pointés) $X_{i}$. 
THÉORÈME 2.4. Soit $\bar{L}=[0,1]$ un treillis fini, $L=(0,1)$ et a un pivot de $L$. Alors $|L|$ a le type d'homotopie de $V_{c \in a^{\perp}}\left(\sum\left(\left|(0, a)^{c}\right| *\left|(0, c)^{a}\right|\right)\right)$.

Le premier ingrédient pour la preuve de ce théorème est la proposition suivante, qui décrit le type d'homotopie d'un produit:

PROPOSITION 2.5. Soit $\bar{E}$ et $\bar{F}$ deux ensembles ordonnés possèdant chacun un plus petit élément 0 et un plus grand élément 1 . Soit $E=\bar{E}-\{0,1\}, F=\vec{F}-\{0,1\}$ et $G=(\tilde{E} \times \vec{F})-\{(0,0),(1,1)\}$. Alors $|G|$ a le type d'homotopie de $\sum(|E| *|F|)$.

Preuve. Soit $X=\{(a, b) \in G ; a<1\}$ et $Y=\{(a, b) \in G ; b<1\}$. Alors $|X|$ a le type d'homotopie d'un cône de sommet $(0,1)$ sur $|X \cap Y|$ car:

$$
(a, b) \leqq(a, 1) \geqq(0,1)
$$

De même, $|Y|$ a le type d'homotopie d'un cône de sommet $(1,0)$ sur $|X \cap Y|$. Non seulement $X \cup Y=G$, mais on a même que $|G|=|X| \cup|Y|$ si bien que $|G|$ a le type d'homotopie de la suspension de $|X| \cap|Y|=|X \cap Y|$. Il suffit donc de montrer que $|X \cap Y|$ est égal à $|E| *|F|$.

Soit $U=\{(a, b) \in X \cap Y ; a>0\}$ et $V=\{(a, b) \in X \cap Y ; b>0\}$. On $a: U \cup V=$ $X \cap Y$ et $|U| \cup|V|=|X \cap Y|$. De plus, $U=E \times(\widetilde{F}-\{1\})$ et donc $|U|=|E| \times C|F|$. De même, $|V|=C|E| \times|F|$. Finalement, $|U| \cap|V|=|U \cap V|=|E \times F|=|E| \times|F|$. Par définition du joint, on a donc $|X \cap Y|=|E| *|F|$.

Remarque. Le fait que $|X \cap Y|=|E| *|F|$ apparaît déjà dans $[Q, \S 1]$.

COROLLAIRE 2.6. $\left|L_{c}\right|$ a le type d'homotopie de $\sum\left(\left|(0, a)^{c}\right| *\left|(0, c)^{a}\right|\right)$.

Preuve. Par le lemme 2.2, $\bar{L}_{\mathrm{c}} \cong[0, a]^{c} \times[0, c]^{a}$ et la proposition précédente d'applique.

Ainsi, pour démontrer le Théorème 2.4 , il nous reste à voir que dans le cas où a est un pivot, $|L|$ a le type d'homotopie de $V_{c \in a^{\perp}}\left|L_{c}\right|$.

PROPOSITION 2.7. Soit $\bar{L}=[0,1]$ un treillis fini, $L=(0,1)$ et $a \in L$. Pour $b, c \in a^{\perp}$, on définit $\bar{L}_{b, c}^{\prime}=\left\{x \in \bar{L} ; x=x_{a} \vee x_{c}, x_{a} \in[0, a]^{b}, x_{c} \in[0, c]\right\}$ et $L_{b, c}^{\prime}=$ $\bar{L}_{b, c}^{\prime}-\{0,1\}$. Alors, l'inclusion $\bigcup_{b, c \in a^{+}} L_{b, c}^{\prime} \hookrightarrow L$ est une équivalence d'homotopie.

Preuve de théorème 2.4. Si $a^{\perp}=\varnothing$, alors $L$ est contractile (Proposition 1.8). Par ailleurs, un bouquet indexé par un ensemble vide est un point. On peut donc 
supposer $a^{\perp} \neq \varnothing$. Comme a est un pivot, on a $[0, a]^{b}=[0, a]^{c}$ si $b, c \in a^{\perp}$ et donc, $L_{b, c}^{\prime}=L_{c, c}^{\prime}=L_{c}^{\prime}$. Par conséquent, l'inclusion $L^{\prime}=\bigcup_{c \in a^{+}} L_{c}^{\prime} \hookrightarrow L$ est une équivalence d'homotopie (Proposition 2.7). La troisième condition définissant un pivot affirme exactement que tout simplexe de $\left|L^{\prime}\right|$ est contenu dans un $\left|L_{c}^{\prime}\right|$. Donc, $\left|L^{\prime}\right|=\bigcup_{c \in a^{+}}\left|L_{c}^{\prime}\right|$. Ainsi, pour compléter la preuve du théorème, il nous reste, compte tenu du Corollaire 2.6, à démontrer les deux assertions suivantes:

a) L'inclusion $i: L_{c} \rightarrow L_{c}^{\prime}$ est une équivalence d'homotopie.

b) $\left|L^{\prime}\right|$ a le type d'homotopie du bouquet $V_{c \in a^{+}}\left|L_{c}^{\prime}\right|$.

a) Par le Théorème $A$ et la Remarque 1.5, il suffit de montrer que si $y \in L_{c}^{\prime}-L_{c}$, alors $y \backslash i=\left\{x \in L_{c} ; x \geqq y\right\}$ est contractile. On a:y=y $y_{a} \vee y_{c}$ avec $y_{a} \in$ $[0, a]^{c}$ et $y_{c} \in[0, c]$. De plus, par le Lemme 2.2 et la Remarque 2.3, on a: $y_{a}=y \wedge a$ et on peut supposer que $y_{c}=y \wedge c$.

Montrons que $y_{a} \vee c \neq 1$. Comme $y_{a} \in[0, a]^{c}, y_{a}=z \wedge a$ avec $z \geqq c$. Or, $z \neq 1$ sinon $y_{a}=a, y \geqq a$ et par conséquent $y_{c}=y \wedge c \in[0, c]^{a}$ contrairement à l'hypothèse $y \notin L_{c}$. Ainsi, $y_{a} \vee c \leqq z<1$. Il s'ensuit que $y_{a} \vee c \in y \backslash i$.

Finalement, $y \backslash i$ a le type d'homotopie d'un cône de sommet $y_{a} \vee c$, car pour $x=x_{a} \vee x_{c} \in y \backslash i$, on $a: x \geqq y_{a} \vee x_{c} \leqq y_{a} \vee c$. A noter que l'application $x \mapsto y_{a} \vee x_{c}$ est bien définie car $x_{c}=x \wedge c$ (Lemme 2.2).

b) On a besoin du lemme suivant:

LEMME 2.8. Une réunion finie de complexes simpliciaux $\bigcup_{i \in I} X_{i}$ a le type d'homotopie du bouquet $V_{i \in I} X_{i}$ si pour tout $J \subset I$ avec card $(J) \geqq 2$, on a $\bigcap_{j \in J} X_{j}$ contractile.

Preuve. On procède par induction sur le cardinal de $I$, le cas card $(I)=2$ étant clair. Soit $k \in I$ et $J=I-\{k\}$. Par hypothèse d'induction, $\bigcup_{i \in J} X_{j}$ est un bouquet et donc pour montrer que $\left(\bigcup_{i \in J} X_{i}\right) \cup X_{k}$ est un bouquet, il suffit de montrer que $\left(\bigcup_{i \in J} X_{j}\right) \cap X_{k}$ est contractile, car $\bigcap_{n \in J \cup\{k\}} X_{n}$ est contractile, donc connexe par arcs. Or $\left(\bigcup_{j \in J} X_{j}\right) \cap X_{k}=\bigcup_{j \in J}\left(X_{j} \cap X_{k}\right)$ est un bouquet de complexes simpliciaux contractiles par hypothèse d'induction, donc contractile.

Appliquons ce lemme à $\left|L^{\prime}\right|=\bigcup_{c \in a^{\perp}}\left|L_{c}^{\prime}\right|$. Soit $J \subset a^{\perp}$ avec card $(J) \geqq 2$. Il s'agit de montrer que $T=\bigcap_{c \in J} L_{c}^{\prime}$ est contractile. Soit $Z=\{x \in T ; a \vee x<1\}$. Si $x \in Z$, alors $a \vee x \in Z$. En effet, il s'agit de voir que $a \vee x \in L_{c}^{\prime}$ pour tout $c \in J$. Or $x=x_{a} \vee x_{c}$ avec $x_{a} \in[0, a]^{c}$ et $x_{c} \in[0, c]$ et donc $a \vee x=a \vee x_{c}$, ce qui démontre que $a \vee x \in$

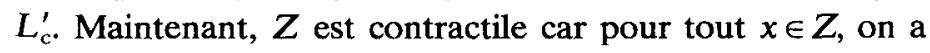

$$
x \leqq a \vee x \geqq a \quad \text { et } \quad a \vee x, a \in Z
$$


Ainsi, pour montrer que $T$ est contractile, il suffit de montrer que l'inclusion $i: Z \hookrightarrow T$ est une équivalence d'homotopie. A cet effet, il suffit (par 1.4 et 1.5) de montrer que pour $y \in T-Z, i / y=\{x \in Z ; x \leqq y\}$ est contractile. Comme $y \notin Z$, $a \vee y=1$. Si $y_{a}=a \wedge y=0$, alors $y$ est un complément de $a$, et de plus pour $c \in J$, $y \in L_{c}^{\prime}$, c'est-à-dire $y=y_{a} \vee y_{c}=y_{c} \in[0, c]$. Par la deuxième condition définissant un pivot, on a $y=c$ et donc $J=\{y\}$ contrairement à l'hypothèse card $(J) \geqq 2$. Ainsi $y_{a}=a \wedge y>0$ et donc $y_{a} \in Z$. Finalement, $i / y$ est contractile car pour tout $x \in i / y$,

$x \leqq y_{a} \vee x \geqq y_{a} \quad$ et $\quad y_{a} \vee x \in i / y$ comme on le vérifie facilement.

Ceci achève la preuve de l'assertion b) et donc du Théorème 2.4

Preuve de la proposition 2.7.

a) Soit $L_{1}=\{x \in L ; x \wedge a$ possède un complément dans [0, $\left.x]\right\}$. Alors l'inclusion $L_{1} \hookrightarrow L$ est une équivalence d'homotopie. En effect, si $y \in L-L_{1}$, alors $(0, y)$ est contractile (Proposition 1.8) et la Proposition 1.6 s'applique.

La suite de la preuve se fait par applications successives du théorème $\mathrm{A}$ et de la remarque 1.5 .

b) Soit $L_{2}=\left\{x \in L_{1}\right.$ tel qu'il existe $b \in a^{\perp}$ avec $\left.x \wedge a \in[0, a]^{b}\right\}$. Alors l'inclusion $i: L_{2} \hookrightarrow L_{1}$ est une équivalence d'homotopie. La preuve est analogue à celle de l'assertion a) dans la démonstration du Théorème 2.4. Soit $y \in L_{1}-L_{2}$ et $y \backslash i=$ $\left\{x \in L_{2} ; x \geqq y\right\}$. Comme $y \in L_{1}, y=(y \wedge a) \vee \bar{y}$ où $\bar{y}$ est un complément de $y \wedge a$ dans $y$. On montre d'abord que $a \vee \bar{y} \neq 1$. Si $a \vee \bar{y}=1, \bar{y}$ est un complément de a ( $\operatorname{car} \bar{y} \wedge a=\bar{y} \wedge y \wedge a=0$ ). Comme $y \in[\bar{y}, 1]$, on en déduit que $y \wedge a \in[0, a]^{\bar{y}}$ et donc $y \in L_{2}$, une contradiction. Ainsi, $a \vee \bar{y}<1$ et $a \vee \bar{y} \in y \backslash i$ comme on le voit facilement. Maintenant $y \backslash i$ est contractile car pour $x \in y \backslash i$, on a:

$$
x \geqq(x \wedge a) \vee \bar{y} \leqq a \vee \bar{y}
$$

Pour rendre ce raisonnement licite, il reste à montrer que $z=(x \wedge a) \vee \bar{y} \in y \backslash i$. D'une part, $x \wedge a \geqq y \wedge a$, et donc $z=(x \wedge a) \vee \bar{y} \geqq(y \wedge a) \vee \bar{y}=y$ et d'autre part, $z \in L_{2}$ car $z \wedge a=x \wedge a \in[0, a]^{b}$ pour un $b \in a^{\perp}$ vu que $x \in L_{2}$. En effet, $x \wedge a \leqq$ $z \wedge a$ car $x \wedge a \leqq z$, et $x \wedge a \geqq z \wedge a$ car $x \geqq z$.

c) Soit $E=\left\{x \in L_{2} ; x \wedge a=0\right.$ et pour tout $\left.c \in a^{\perp}, x \notin[0, c]\right\}$. Alors l'inclusion $i: L_{3}=L_{2}-E \hookrightarrow L_{2}$ est une équivalence d'homotopie. Soit $y \in E$ et $y \backslash i=\{x \in$ $\left.L_{3} ; x \geqq y\right\}$. On a: $a \vee y \neq 1$, sinon $y \in a^{\perp}$ et $y \in[0, y]$ contrairement au fait que $y \in E$. Maintenant, $y \backslash i$ est contractile car pour $x \in y \backslash i$, on a:

$$
x \geqq(x \wedge a) \vee y \leqq a \vee y .
$$


Ceci est licite, car $(x \wedge a) \vee y \in y \backslash i$. En effet, si $x \wedge a=0, x \in[0, c]$ pour un $c \in a^{\perp}$ car $x \in L_{3}$ et $y \leqq x \leqq c$ ce qui est contraire à $y \in E$. Donc $x \wedge a \neq 0$ et $(x \wedge a) \vee y \in$ $L_{3}$.

d) Soit $L_{4}=\bigcup_{b, c \in a^{\perp}} L_{b, c}^{\prime}$. Alors l'inclusion $i: L_{4} \hookrightarrow L_{3}$ est une équivalence d'homotopie. Soit $y \in L_{3}-L_{4}$ et soit $\bar{y}$ un complément de $y \wedge a$ dans $y$. Si $y \wedge a=0, y=\bar{y} \in[0, c]$ pour un $c \in a^{\perp}\left(\right.$ car $\left.y \in L_{3}=L_{2}-E\right)$ et donc $y=0 \vee \bar{y} \in L_{c, c}^{\prime}$ contrairement au fait que $y \notin L_{4}$. Ainsi, $y \wedge a>0$ et $y \wedge a \in i / y=\left\{x \in L_{4} ; x \leqq y\right\}$. On notera en effet que $y \wedge a \in[0, a]^{b}$ pour un $b \in a^{\perp}$ car $y \in L_{2}$. Maintenant, $i / y$ est contractile car pour $x \in i / y$, on $a$ :

$$
x \leqq(y \wedge a) \vee x \geqq y \wedge a
$$

et il reste à montrer que $z=(y \wedge a) \vee x \in i / y$. Clairement, $y \wedge a \leqq z \wedge a$ car $y \wedge a \leqq$ $z$, et $y \wedge a \geqq z \wedge a$ car $y \geqq z$. Donc, $z \wedge a=y \wedge a$. Par ailleurs comme $x \in L_{4}$, $x=x_{a} \vee x_{c}$ avec $x_{a} \leqq a$ et $x_{c} \leqq c$. Comme $x_{a} \leqq x \wedge a \leqq y \wedge a$, on $a: z=(y \wedge a) \vee x_{c} \in$ $L_{b, c}^{\prime}$. Ainsi, $z \in L_{4}$ et donc $z \in i / y$.

\section{Treillis semi-modulaires et modulaires}

Afin de pouvoir appliquer le Théorème 2.4, il est nécessaire de mettre d'abord la main sur un pivot dans le treillis qui nous intéresse. La proposition ci-dessous fait un pas dans cette direction pour les treillis semi-modulaires et résout la question pour les treillis modulaires. Auparavant, rappelons qu'un treillis $\bar{L}$ est semi-modulaire (supérieurement) si:

pour $a, b \in \bar{L}$ avec $a \wedge b$ maximal dans $a$, alors $b$ est maximal dans $a \vee b$.

Dans un treillis semi-modulaire fini, la longueur de toutes les chaînes entre 0 et $x$ est constante, et on la note $r(x)$. De plus, la fonction $r$ satisfait:

$$
r(x \vee y)+r(x \wedge y) \leqq r(x)+r(y)
$$

Un treillis est modulaire si à la fois $\bar{L}$ et le treillis avec ordre opposé sont semi-modulaires. Dans ce cas, l'inégalité satisfaite par la fonction $r$ devient une égalité. Notons que la définition usuelle de treillis modulaire est la suivante:

$$
\text { pour } a, b, c \in \bar{L}, \text { si } c \leqq a, \text { alors } a \wedge(b \vee c)=(a \wedge b) \vee c
$$

Enfin, un treillis fini $\tilde{L}$ est dit géométrique s'il est semi-modulaire et si tout point 
de $\bar{L}-\{0\}$ est le supremum d'une famille d'éléments minimaux (non nuls) de $\bar{L}$. Pour plus de détails sur ces définitions, voir [A, chap. II].

PROPOSITION 3.1. Soit $\bar{L}=[0,1]$ un treillis semi-modulaire fini et $a \in \bar{L}$.

a) Si $c \in a^{\perp}$ satisfait $r(a)+r(c)=r(1)$, alors $[0, c]^{a}=[0, c]$ et $[0, a]^{c}=[0, a]$.

b) Si $r(a)+r(c)=r(1)$ pour tout $c \in a^{\perp}$, alors deux compléments distincts de a ne sont pas comparables.

c) Si a est maximal et si $r(a)+r(c)=r(1)$ pour tout $c \in a^{1}$, alors a est un pivot.

d) Si $\bar{L}$ est modulaire et si a est maximal, alors a est un pivot.

COROLLAIRE 3.2. Dans les hypothèses c) ou d) de la proposition 3.1, et si $L=(0,1)$ on $a$ :

$|L|$ a le type d'homotopie de $V_{\mathrm{c} \in a^{\perp}}\left(\sum|(0, a)|\right)$.

Preuve. Par la partie a) de la proposition, $(0, a)^{c}=(0, a)$. De plus, $(0, c)=\varnothing$ car la condition $r(a)+r(c)=r(1)$ implique que $c$ est minimal. Ainsi,

$$
|(0, a)| *|(0, c)|=|(0, a)|
$$

et on conclut par le Théorème 2.4 .

Preuve de la Proposition 3.1. a) Si $y \in[0, c]$, il est clair que $(y \vee a) \wedge c \geqq y$. Par ailleurs, $r((y \vee a) \wedge c)+r(1) \leqq r(y \vee a)+r(c)$ par semi-modularité et le fait que $(y \vee a) \vee c=a \vee c=1$. De plus, $r(y \vee a) \leqq r(y)+r(a)$ par semi-modularité et le fait que $y \wedge a=0$. On en déduit $r((y \vee a) \wedge c)+r(1) \leqq r(y)+r(a)+r(c)$ et donc

$$
r((y \vee a) \wedge c) \leqq r(y)
$$

grâce à l'hypothèse. Cela force alors l'égalité $(y \vee a) \wedge c=y$. La deuxième assertion résulte de l'échange des rôles de $a$ et $c$.

b) L'hypothèse implique que $r(c)$ est constant pour $c \in a^{\perp}$. Donc, si $b, c \in a^{\perp}$ et $b \leqq c$, alors $b=c$.

c) Les deux premières conditions de la définition d'un pivot résultent de a) et b). Compte tenu de a), et du Lemme 2.2 , on a:

$$
\vec{L}_{c}=\bar{L}_{c}^{\prime}=\left\{x \in \bar{L} ; x=x_{a} \vee x_{c}, x_{a}=x \wedge a, x_{c}=x \wedge c\right\}
$$

Comme a est maximal et $r(a)+r(c)=r(1)$, on a $r(c)=1$, et donc $x_{c}$ ne peut valoir que 0 ou c. Les éléments $x$ tels que $x_{c}=0$ appartiennent à tous les $L_{c}$ et ne jouent 
donc aucun rôle pour la vérification de la troisième condition de la définition d'un pivot. En fait, pour vérifier cette condition, il suffit de montrer que si $x \in L_{c}$ avec $x_{c}=c, y \in L_{d}$ avec $y_{d}=d$ et $x \leqq y$, alors $y \in L_{c}$.

Comme $c \leqq x \leqq y$, on a: $y_{a} \vee c \leqq y$ et il suffit de montrer l'égalité. Comme $y_{a}<y_{a} \vee c \leqq y$, il suffit de voir que $y_{a}$ est maximal dans $y$. Or cela est immédiat par semi-modularité car 0 est maximal dans $d, y_{a} \wedge d=0$ et $y_{a} \vee d=y$.

d) $\mathrm{Si} \bar{L}$ est modulaire, la condition $r(a)+r(c)=r(1)$ est toujours satisfaite pour $c \in a^{\perp}$. On conclut donc à l'aide de c).

Remarque. Si a est minimal, alors par semi-modularité, tout $c \in a^{\perp}$ est maximal. La condition $r(a)+r(c)=r(1)$ est donc automatique. Pour que a soit un pivot, il faudrait encore que la troisième condition soit satisfaite, ce qui n'est pas clair en général.

PROBLÈME 3.3. Dans un treillis semi-modulaire, existe-t-il toujours un élément minimal qui soit un pivot?

THÉORÈME 3.4. Soit $\bar{L}=[0,1]$ un treillis modulaire fini et $L=(0,1)$. Soit $0=a_{0}<a_{1}<\cdots<a_{n-1}<a_{n}=1$ une chaíne maximale dans $\bar{L}$. Soit $m_{i}$ le nombre de compléments de $a_{i}$ dans $\left[0, a_{i+1}\right]$ et $m=\prod_{i=0}^{n-1} m_{i}$. Alors $L$ a le type d'homotopie d'un bouquet de $m$ sphères de dimension $n-2$.

Preuve. Par induction sur $n$. Si $n=1, m=m_{0}=1, L=\varnothing$ et $|L|=S^{-1}$. Si $n>1$, on applique le Corollaire 3.2 avec $a=a_{n-1}$ et on conclut en appliquant l'hypothèse d'induction à $\left(0, a_{n-1}\right)$.

Remarques. 1) En renversant l'ordre du treillis, on voit que $L$ a aussi le type d'homotopie d'un bouquet de $s=\prod_{i=1}^{n} s_{i}$ sphères de dimension $n-2$ où $s_{i}$ est le nombre de compléments de $a_{i}$ dans $\left[a_{i-1}, 1\right]$. On a donc: $m=s$.

2) Le nombre $m$ est égal (au signe près) à la caractéristique d'Euler réduite $\tilde{\chi}(L)=\chi(L)-1$. Or il est bien connu $[\mathrm{R}$, thm 3$]$ que $\tilde{\chi}(L)=\mu(0,1)$ où $\mu$ est la fonction de Möbius de $L$. Une formule dûe à Crapo (voir [A, p. 170]) exprime $\mu(0,1)$ en termes de $\mu(0, c)$ et $\mu(c, 1)$ (égal ici à $\mu(0, a)$ ). Une application répétée de cette formule donne aisément $\tilde{\chi}(L)=(-1)^{n} \prod_{i=0}^{n-1} m_{i}$.

3) Si la solution du Problème 3.3 est positive, on montre de manière analogue que si $\bar{L}$ est semi-modulaire, alors $|L|$ a le type d'homotopie d'un bouquet de sphères de dimension $n-2$ où $n=r(1)$. De toute façon, ce dernier résultat semble problable car Folkman $[\mathrm{F}]$ a montré qu'un treillis géométrique a le type d'homologie d'un bouquet de sphères de dimension $n-2$. Or tout treillis fini 
semi-modulaire a le type d'homotopie d'un treillis géométrique. En effet:

PROPOSITION 3.5. Soit $\bar{L}=[0,1]$ un treillis fini semi-modulaire et $L=(0,1)$. Soit $\psi: \bar{L} \rightarrow \bar{L}$ défini par: $\psi(x)$ est le supremum de tous les éléments minimaux de $[0, x]$. Alors:

a) $\bar{E}=\psi(\bar{L})$ est un treillis géométrique.

b) Si $E=\psi(L)$, alors $\psi: L \rightarrow E$ est une équivalence d'homotopie.

Preuve. a) Si $a, b \in \bar{E}, a \vee b \in \bar{E}$. Par ailleurs, on définit $a \cap b=\psi(a \wedge b)$. On vérifie aisément que $\bar{E}$ est un treillis pour $\vee$ et $\cap$. (En fait, on peut montrer que $a \cap b=a \wedge b$, mais cela ne joue pas de rôle ici). Par construction, tout point de $\bar{E}$ est le supremum d'éléments minimaux. Il reste donc à montrer que $\bar{E}$ est semi-modulaire. Si, $a, b \in \bar{E}$ et si $a \cap b$ est maximal dans $a$ (dans $\bar{E}$ ), alors il existe $m$ minimal avec $m \leqq a$ et $m \leqq b$ (sinon, $a \leqq b$ et $a \cap b=a \wedge b=a$ ). Donc $(a \cap b)<$ $(a \cap b) \vee m \leqq a$ et par conséquent $(a \cap b) \vee m=a$. Maintenant $m$ est minimal, $b \wedge m=0$ et donc par semi-modularité de $\bar{L}, b$ est maximal dans $m \vee b$. Or $m \vee b \leqq a \vee b$ car $m \leqq a$ et $m \vee b \geqq a \vee b$ car $m \vee b \geqq b$ et $m \vee b \geqq m \vee(a \cap b)=a$. Ainsi $b$ est maximal dans $a \vee b=m \vee b$.

b) est clair, car si $i: E \rightarrow L$ est l'inclusion, alors $\psi \circ i=\mathrm{id}_{E}$ et $i \circ \psi$ est homotope à id $\mathrm{id}_{\mathrm{L}}$ car $\psi(x) \leqq x$ pour tout $x \in L$.

Comme application du Théorème 3.4 , voici la version homotopique d'un résultat homologique dû à Lusztig [L, chap. 1]:

PROPOSITION 3.6. Soit $V$ un espace vectoriel de dimension $n$ sur un corps fini $\grave{a} q$ éléments. Soit $\bar{L}$ le treillis des sous-espaces de $V$ et $L=(0, V)$. Alors $L$ a le type d'homotopie d'un bouquet de $q^{\left(\frac{n}{2}\right)}$ sphères de dimension $n-2$.

Preuve. En vertu du Théorème 3.4, il suffit de calculer le nombre $m_{i}$ de compléments d'un sous-espace $W_{i}$ de dimension $i$ dans un espace de dimension $i+1$. Or le nombre de sous-espaces de dimension 1 de $W_{i+1}$ est égal à $s=$ $\left(q^{i+1}-1\right) /(q-1)$ et le nombre de ces sous-espaces qui sont contenus dans $W_{i}$ est $t=\left(q^{i}-1\right) /(q-1)$. Donc le nombre de compléments de $W_{i}$ dans $W_{i+1}$ est $m_{i}=$ $s-t=q^{i}$. Par conséquent, le nombre de sphères du bouquet est

$$
m=\prod_{i=0}^{n-1} m_{i}=q^{\sum_{t=0}^{n-1} i}=q^{\left(\frac{n}{2}\right)}
$$




\section{Le treillis des sous-groupes d'un groupe fini}

Soit $G$ un groupe fini, $\bar{L}(G)$ le treillis des sous-groupes de $G$ et $L(G)=(1, G)$. On s'intéresse d'abord à trouver des conditions pour que $L(G)$, ou plus généralement $(H, G)$ soit contractile ( $H$ étant un sous-groupe de $G$ ).

Si $N$ est un sous-groupe normal de $G$, la notion de complément de $N$ en théorie des groupes coïncide avec celle introduite pour les treillis. Ainsi, par la proposition $1.8, L(G)$ est contractile s'il existe un sous-groupe normal sans complément. Un exemple typique d'un tel sous-groupe est le sous-groupe de Frattini $\Phi(G)$, s'il est non trivial. Plus généralement, soit $\Phi(H, G)$ l'intersection de tous les sous-groupes maximaux de $G$ contenant $H$. Ainsi $\Phi(G)=\Phi(1, G)$.

LEMME 4.1. Si $\Phi(H, G) \neq H$, alors $(H, G)$ est contractile.

Preuve. Si $K \in(H, G), \Phi \vee K<G$ où $\Phi \vee K$ désigne le sous-groupe engendré par $\Phi=\Phi(H, G)$ et $K$. Par conséquent,

$K \leqq \Phi \vee K \geqq \Phi$.

PROPOSITION 4.2. Soit $N$ un sous-groupe normal de $G, H$ un sous-groupe de $G$ et $X=N \cdot H$. Si $(X, G)$ est contractile, alors $(H, G)$ l'est aussi. En particulier, $L(G)$ est contractile si $L(G / N)$ l'est.

Remarque. Dans le même ordre d'idée, on démontre aisément que si $M \in$ $(X, G)$ n'a pas de complément dans $(X, G)$, alors il n'en a pas dans $(H, G)$.

Preuve. On procède par induction sur l'ordre de $G$. Soit $A=$ $\{Z \in(H, G) ; Z \cdot N=G\}$. Soit $Z \in A$ et $X^{\prime}=(Z \cap N) \cdot H$. Montrons tout d'abord que $[X, G] \rightarrow\left[X^{\prime}, Z\right] ; U \mapsto U \cap Z$ et $\left[X^{\prime}, Z\right] \rightarrow[X, G] ; V \mapsto V \cdot N$ sont des bijections réciproques. D'une part $(U \cap Z) \cdot N=U \cap(Z \cdot N)$ car $U \geqq N$ et donc $(U \cap Z) \cdot N=U \cap G=U$. D'autre part, $Z \cap(V \cdot N)=V \cdot(Z \cap N)$ car $V \leqq Z$ et donc $Z \cap(V \cdot N)=V$ car $Z \cap N \leqq X^{\prime} \leqq V$.

Par hypothèse $(X, G)$ est contractile et donc $\left(X^{\prime}, Z\right)$ l'est aussi. L'hypothèse d'induction appliquée à $Z$ implique que $(H, Z)$ est contractile. A noter qu'au départ de l'induction, l'ensemble $A$ est nécessairement vide. Par la Proposition 1.6, $(H, G)-A \hookrightarrow(H, G)$ est une équivalence d'homotopie. Or $(H, G)-A$ est contractile, car pour $T \in(H, G)-A$, on a:

$$
T \leqq T \cdot N \geqq X
$$

Ainsi $(H, G)$ est contractile. Le cas particulier résulte du cas $H=1$. 
COROLLAIRE 4.3. Soit $N$ et $M$ deux sous-groupes normaux de $G$ avec $N<M$. Si $\Phi(N, M) \neq N$, alors $L(G)$ est contractile.

Preuve. Soit $Z \in(N, G)$ maximal et posons $\Phi=\Phi(N, M)$. Si $\Phi \cdot Z=G$, alors $\Phi \cdot(Z \cap M)=M$ et donc $Z \cap M=M$. Par conséquent, $Z \geqq M \geqq \Phi$ et $G=\Phi \cdot Z=$ $Z$, ce qui est impossible. Donc $\Phi \cdot Z=Z$ par maximalité de $Z$ et $\Phi \leqq Z$. Finalement, $\Phi \leqq \Phi(N, G)$ ce qui montre que $\Phi(N, G) \neq N$. Par le Lemme 4.1, $(N, G)$ est contractile et par la Proposition 4.2, $L(G)$ l'est aussi.

Nous allons étudier $L(G)$ pour $G$ nilpotent, puis résoluble. Mais auparavant, notons encore ce cas particulier de la Proposition 2.5:

PROPOSITION 4.4. Soit $G$ et $H$ deux groupes d'ordres premiers entre eux. Alors, $|L(G \times H)|$ a le type d'homotopie de $\sum(|L(G)| *|L(H)|)$.

Preuve. Par la Proposition 2.5, il suffit de montrer que $\tilde{L}(G \times H)=$ $\bar{L}(G) \times \bar{L}(H)$. Mais ceci est clair pour des groupes d'ordres premiers entre eux.

PROPOSITION 4.5. Soit $G$ un groupe nilpotent et $H$ un sous-groupe.

a) Si $H$ n'est pas normal dans $G,(H, G)$ est contractile.

b) Si $H \triangleleft G$ et $G / H$ n'est pas un produit de groupes abéliens élémentaires, alors $(H, G)=L(G / H)$ est contractile.

c) Si $H \triangleleft G$ et $G / H \cong \prod_{i=1}^{r} C_{p_{1}}^{n_{1}}$, alors $|(H, G)|=|L(G / H)|$ a le type d'homotopie d'un bouquet de $m$ sphères de dimension $n-2$ où $m=\prod_{i=1}^{r} p_{i}^{\left(\eta_{i}\right)}$ et $n=\sum_{i=1}^{r} n_{i}$. $\left(C_{p}^{n}\right.$ désigne le produit de n copies du groupe cyclique d'ordre premier $p$ ).

Preuve. a) Comme $G$ est nilpotent, tout sous-groupe maximal de $G$ est normal et par conséquent $\Phi(H, G) \triangleleft G$. Donc $\Phi(H, G) \neq H$ et $(H, G)$ est contractile par le Lemme 4.1.

b) Comme $G$ est nilpotent, $G / \Phi(H, G)$ est un produit de groupes abéliens élémentaires. Donc $\Phi(H, G) \neq H$ et $(H, G)$ est contractile.

c) On peut supposer $H=1$. Soit $P_{i}=C_{p_{i}}^{n_{i}}$ le $p_{i}$-sous-groupe de Sylow de $G$. Par la Proposition 3.6, $\left|L\left(P_{i}\right)\right|$ a le type d'homotopie d'un bouquet de $\left.p\right|_{i} ^{\left(\eta_{i}\right)}$ sphères de dimension $n_{i}-2$. Comme les $\boldsymbol{P}_{i}$ sont d'ordres deux à deux premièrs entre eux, $1 \mathbf{a}$ Proposition 4.4 donne la recette du calcul de $|L(G)|$.

Le problème étant résolu pour les groupes nilpotents, on va s'intéresser aux groupes résolubles. Tout d'abord, le lemme suivant justifie la notation $[1, N]^{H}$ introduite pour des treillis quelconques. 
LEMME 4.6. Soit $N$ un sous-groupe normal de $G$ et $H$ un complément de $N$.

a) $[1, N]^{H}$ est le treillis des sous-groupes de $N$ qui sont invariants par $H$.

b) Si $N$ est abélien, $[1, N]^{H}$ est le treillis des sous-groupes de $N$ qui sont normaux dans $G$.

c) L'application $[H, G] \rightarrow[1, N]^{H}, X \mapsto X \cap N$ est un isomorphisme de treillis. En particulier, un complément d'un sous-groupe normal abélien minimal est maximal.

d) L'application $[N, G] \rightarrow[1, H], X \mapsto X \cap H$ est un isomorphisme de treillis et donc $[1, H]^{N}=[1, H]$.

e) Si $N$ est un sous-groupe normal abélien minimal et si $N \cdot K=G$ avec $K \neq G$, alors $K$ est un complément de $N$.

Preuve. Soit $T$ le treillis des sous-groupes de $N$ qui sont invariants par $H$. Si $X \in[H, G]$, alors $X \cap N \in T$ car $X \cap N$ est normalisé par $X$, donc par $H$. Considérons les applications $\phi:[H, G] \rightarrow T, X \mapsto X \cap N$ et $\psi: T \rightarrow[H, G], Y \mapsto$ $Y \cdot H$. Comme $H$ normalise $Y \in T, Y \cdot H$ est bien un sous-groupe. Ces applications sont inverses l'une de l'autre car:

$$
\begin{aligned}
& \text { comme } H \leqq X,(X \cap N) \cdot H=X \cap N \cdot H=X \cap G=X \\
& \text { comme } Y \leqq N, Y \cdot H \cap N=Y \cdot(H \cap N)=Y \cdot 1=Y
\end{aligned}
$$

Par définition, $[1, N]^{H}$ est l'image de $\phi$ et donc $[1, N]^{H}=T$, ce qui démontre a) et c). Si $N$ est abélien et $Y \in[1, N]^{H}$, alors $N$ normalise $Y$ et donc $Y$ est normal dans $N \cdot H=G$, ce qui prouve b). Le cas particulier de c) en résulte car alors $(H, G)=\varnothing$. Pour montrer d), considérons les applications $\alpha:[N, G] \rightarrow[1, H]$, $X \mapsto X \cap H, \beta:[1, H] \rightarrow[1, G / N], Y \mapsto Y \cdot N / N$ et $\gamma:[N, G] \rightarrow[1, G / N], X \mapsto$ $X / N$. Clairement, $\beta$ et $\gamma$ sont des isomorphismes de treillis et donc $\alpha$ en sera un si l'on montre que $\beta^{\circ} \alpha=\gamma$. Mais ceci est clair car pour $X \geqq N$, on a:

$$
(X \cap H) \cdot N=X \cap H \cdot N=X \cap G=X
$$

Pour montrer e), on remarque que $K \cap N$ est normalisé par $K$ et par $N$, donc par $G$. Comme $K \neq G, K \cap N \neq N$ et donc $K \cap N=1$ par minimalité de $N$.

La technique des pivots mise en place au paragraphe 2 va s'appliquer au cas des groupes résolubles grâce au résultat suivant:

THÉORÈME 4.7. Soit $N$ un sous-groupe normal abélien de $G$. On suppose que $N$ est semi-simple comme $\mathbb{Z} G$-module ( $G$ agissant par conjugaison sur $N$ ). Alors $N$ est un pivot de $\ddot{L}(G)$. 
Preuve. Il n'y a rien à vérifier si $N$ n'a pas de complément. Sinon, par le Lemme 4.6b), $[1, N]^{H}$ est indépendant de $H \in N^{\perp}$, ce qui montre la condition (i) de la définition d'un pivot. La condition (ii) est claire, car si deux compléments $H$ et $K$ sont tels que $H \leqq K$, alors $H=K$ vu qu'ils ont le même ordre. Pour la condition (iii), rappelons que $\bar{L}_{H}^{\prime}=\left\{X=M \cdot K ; M \in[1, N]^{H}, K \in[1, H]\right\}$ et $L_{H}^{\prime}=$ $\check{L}_{H}^{\prime}-\{1, G\}$. Soit $H_{1}, \ldots, H_{n}$ des compléments de $N$ et $X_{i} \in L_{H_{r}}^{\prime}(1 \leqq i \leqq n)$. Il s'agit de montrer que si $X_{n} \leqq X_{n-1} \leqq \cdots \leqq X_{1}$, alors tous les $X_{i}$ appartiennent à un même $L_{H}^{\prime}$. C'est trivial si $n=1$, et par induction, on peut supposer que $X_{n}$, $X_{n-1}, \ldots, X_{2} \in L_{H}^{\prime}$. Disons que $X_{1} \in L_{H}^{\prime}$, ce qui permet d'écrire $X_{1}=M_{1} \cdot K_{1}$ et $X_{i}=M_{i} \cdot K_{i}^{\prime}$ avec $K_{1} \leqq H$ et $K_{i}^{\prime} \leqq H^{\prime}(2 \leqq i \leqq n)$. Notons que $M_{n} \leqq M_{n-1} \leqq \cdots \leqq$ $M_{1}$ et que $K_{n}^{\prime} \leqq K_{n-1}^{\prime} \leqq \cdots \leqq K_{2}^{\prime}$.

Tout élément de $H^{\prime}$ s'écrit $h^{\prime}=c(h) \cdot h$ avec $h \in H$ et $c(h) \in N$. En fait, l'application $c: H \rightarrow N$ est un 1-cocycle [H, 17.3]. Au sous-groupe $K_{i}^{\prime}$ de $H^{\prime}$ correspond un sous-groupe $K_{i}$ de $H$ en sorte que $K_{i}^{\prime}=\left\{c(h) \cdot h ; h \in K_{i}\right\}$. Montrons tout d'abord que $c\left(K_{2}\right) \subset M_{1}$. Si $h \in K_{2}, c(h) \cdot h \in K_{2}^{\prime} \leqq M_{1} \cdot K_{1}$ et donc $c(h) \in M_{1}$. Maintenant $M_{1} \in[1, N]^{H}$, donc $M_{1}$ est un sous-groupe normal de $G$, en d'autres termes $M_{1}$ est un sous- $\mathbb{Z} G$-module de $N$. Comme $N$ est semi-simple, $N=M_{1} \times P$ où $P$ est aussi un sous- $\mathbb{Z} G$-module de $N$. On peut donc écrire $c=c_{1} \times c_{2}$ avec $c_{1}: H \rightarrow M_{1}$ et $c_{2}: H \rightarrow P$ des 1 -cocycles. Comme $c\left(K_{2}\right) \subset M_{1}$, on a $\left.c_{2}\right|_{K_{2}}=0$ et $\left.c_{1}\right|_{K_{2}}=\left.c\right|_{K_{2}}$. Soit alors $H^{\prime \prime}=\left\{c_{1}(h) \cdot h ; h \in H\right\}$. Comme $c_{1}$ est un cocyle, $H^{\prime \prime}$ est un sous-groupe de $G$, complément de $N$. Alors pour $i \geqq 2, K_{i}^{\prime \prime}=\left\{c_{1}(h) \cdot h ; h \in K_{i}\right\}=$ $\left\{c(h) \cdot h ; h \in K_{i}\right\}=K_{i}^{\prime}$ car $c_{1}$ et $c$ coïncident sur $K_{i} \leqq K_{2}$. Donc, $X_{i}=M_{i} \cdot K_{i}^{\prime}=$ $M_{i} \cdot K_{i}^{\prime \prime} \in L_{H^{\prime \prime}}^{\prime}$. De plus $M_{1} \cdot K_{1}=M_{1} \cdot K_{1}^{\prime \prime}$ où $K_{1}^{\prime \prime}=\left\{c_{1}(h) \cdot h ; h \in K_{1}\right\}$ car $K_{1}^{\prime \prime} \leqq$ $M_{1} \cdot K_{1}$ vu que $c_{1}$ est à valeurs dans $M_{1}$. Donc $X_{1}=M_{1} \cdot K_{1}^{\prime \prime} \in L_{H^{\prime \prime}}^{\prime}$. Cela démontre la condition (iii) de la définition d'un pivot et achève la preuve du théorème.

Remarque. Des exemples montrent que la condition (iii) de la définition d'un pivot n'est en général pas satisfaite si $N$ n'est pas semi-simple comme $\mathbb{Z} G$ module.

COROLLAIRE 4.8. Soit $N$ un sous-groupe normal abélien de $G$. Alors $|L(G)|$ a le type d'homotopie de $V_{H \in N^{\perp}}\left(\sum\left(\left|(1, N)^{\mathbf{H}}\right| *|L(G / N)|\right)\right)$. De plus $L(G)$ est contractile si $N$ n'est pas semi-simple comme $\mathbb{Z} G$-module.

Preuve. Si $N$ est semi-simple ou si $N$ n'a pas de complément, $N$ est un pivot et le Théorème 2.4 s'applique. On conclut en constatant que $(1, H)^{N}=(1, H) \cong$ $L(G / N)$ en vertu du Lemme $4.6 \mathrm{~d})$.

Si $N$ n'est pas semi-simple, on va montrer que $L(G)$ et $(1, N)^{H}$ sont contractiles, ce qui permet de conclure. Soit $J$ le radical du $\mathbb{Z} G$-module $N$, donc $J \neq 1$. Comme $[1, N]^{H}$ est le treillis des sous- $\mathbb{Z} G$-modules de $N$, un argument identique à celui du Lemme 4.1 montre que $(1, N)^{H}$ est contractile. 
Par ailleurs, $J$ est caractéristique dans $N$, donc normal dans $G$. Comme $N$ possède un complément, $N / J$ aussi. Par conséquent, si $J$ possède un complément $C$, alors $C \cong G / J$ et donc $C$ contient un complément $H$ de $N$. Donc $H$ normalise $C \cap N$ et $C \cap N \in[1, N]^{\mathrm{H}}$. Mais il est clair que $C \cap N$ est alors un complément de $J$ dans $[1, N]^{H}$, ce qui est absurde. Donc, $J$ ne possède pas de complément dans $[1, G]$, et donc $L(G)$ est contractile (Proposition 1.8).

Si $G$ est résoluble, nous allons déterminer le type d'homotopie d'un intervalle ( $H, K)$ de $L(G)$. Comme tout sous-groupe de $G$ est résoluble, il suffit de traiter le cas $K=G$.

Rappelons qu'une suite croissante de sous-groupes de $G$ est dite principale (respectivement caractéristique maximale) si chaque sous-groupe de la suite est normal (respectivement caractéristique) dans $G$ et si la suite ne peut être raffinée en une suite ayant la même propriété.

Soit $1=N_{0} \triangleleft N_{1}<\cdots \triangleleft N_{s}=G$ une suite principale de $G$. Pour un sousgroupe $H$ fixé, soit $H=H_{0} \triangleleft H_{1} \triangleleft \cdots \triangleleft H_{n}=G$ les termes distincts de la suite des $H \cdot N_{j}$. Soit $R_{i}=N_{r_{i}}$ le plus grand des $N_{j}$ tels que $H \cdot N_{j}=H_{i}$ et $L_{i}$ le plus petit des $N_{i}$ tels que $H \cdot N_{j}=H_{i}(0 \leqq i \leqq n)$. Ainsi, $L_{0}=1, N_{r_{i}+1}=L_{i+1}$ et $R_{n}=G$.

THÉORĖME 4.9. Soit $H$ un sous-groupe propre d'un groupe résoluble $G$. Dans les notations ci-dessus, soit $m_{i}$ le nombre de compléments de $L_{i} / R_{i-1}$ dans $G / R_{i-1}$ qui contiennent $H_{i-1}=H \cdot R_{i-1}(1 \leqq i \leqq n)$. Alors $|(H, G)|$ a le type d'homotopie d'un bouquet de $m_{1} \cdot m_{2} \cdots m_{n}$ sphères de dimension $n-2$. De plus $m_{n}=1$.

Preuve. Comme $\boldsymbol{R}_{0} \subset H$, quitte à passer au quotient par $\boldsymbol{R}_{0}$, on peut supposer $R_{0}=1$ si bien que $L_{1}$ est un sous-groupe normal minimal de $G$, en particulier abélien. On procède par induction sur $n$. Si $n=1, H \cdot L_{1}=G$. Par le Lemme 4.6e), $H$ est un complément de $L_{1}$. Par le Lemme 4.6c), $H$ est maximal et donc $|(H, G)|=\varnothing=S^{-1}$. Par ailleurs $m_{1}=1$ (car $H$ est l'unique complément de $L_{1}$ contenant $H$ ) et ceci prouve que $m_{n}=1$ en général.

Si $n>1$, par hypothèse d'induction, $\left|\left(H_{1}, G\right)\right|$ est homotope à un bouquet de $m_{2} \cdots m_{n}$ sphères de dimension $n-3$.

ASSERTION 1. C est un complément de $H_{1}$ dans $[H, G]$ si et seulement si $C$ est un complément de $L_{1}$ dans $[1, G]$ et $C \geqq H$.

ASSERTION 2. $H_{1}$ est un pivot de $[H, G]$. 
Il résulte de l'Assertion 1 que $m_{1}$ est le nombre de compléments de $H_{1}$ dans $[H, G]$ et que chacun de ces compléments est maximal dans $G$ (Lemme 4.6c)). Par ailleurs en vertu de l'Assertion 2, le Théorème 2.4 s'applique:

$$
|(H, G)| \cong V_{C \in H^{\frac{1}{1}}} \sum\left(\left|\left(H, H_{1}\right)^{C}\right| *\left|(H, C)^{H_{1}}\right|\right) .
$$

Maintenant, $\left(H, H_{1}\right)^{C}$ est l'image de $(C, G)$ par intersection avec $H_{1}$, donc vide car $C$ est maximal. De plus $(H, C)^{H_{1}}=(H, C) \cong\left(H_{1}, G\right)$ en restreignant aux intervalles au dessus de $H$ et de $H_{1}$ l'isomorphisme du Lemme 4.6d):

$$
(1, C)^{L_{1}}=(1, C) \cong\left(L_{1}, G\right) .
$$

Ainsi, $|(H, G)|$ est homotope à un bouquet, indexé par les $m_{1}$ compléments de $H_{1}$ dans $[H, G]$, de suspensions de $\left|\left(H_{1}, G\right)\right|$. Le théorème en découle.

Preuve de l'assertion 1. Si $C$ est un complément de $L_{1}$ contenant $H$, alors $C \cdot H_{1}=C \cdot H \cdot L_{1}=G$ et $C \cap H_{1}=C \cap H \cdot L_{1}=H \cdot\left(C \cap L_{1}\right)=H$. Réciproquement, si $C$ est un complément de $H_{1}$ dans $[H, G]$, alors $C \cdot L_{1}=C \cdot H \cdot L_{1}=$ $C \cdot H_{1}=G$ et donc $C$ est un complément de $L_{1}$ en vertu du lemme 4.6e).

Preuve de l'assertion 2. On a vu ci-dessus que $\left(H, H_{1}\right)^{C}$ est vide, donc indépendant de $C$. Comme les compléments de $H_{1}$ sont des compléments de $L_{1}$ dans $[1, G]$, ils ne peuvent être comparables vu que les compléments d'un sous-groupe normal ont tous le même ordre. Il reste donc à montrer la condition (iii) de la définition d'un pivot. Soit $Z_{1} \leqq Z_{2} \leqq \cdots \leqq Z_{m}$ avec $Z_{i}=$ $\left(Z_{i} \cap H_{1}\right) \cdot\left(Z_{i} \cap C_{i}\right)$ pour un $C_{i} \in H_{1}^{\perp}$ et $Z_{i} \cap H_{1} \in\left[H, H_{1}\right]^{C}$. Comme $\left(H, H_{1}\right)^{C}$ est vide, $Z_{i} \cap H_{1}$ ne peut prendre que les valeurs $H$ ou $H_{1}$. Soit $r$ le plus grand indice $i$ tel que $Z_{i} \cap H_{1}=H$ si bien que $Z_{i}=Z_{i} \cap C_{i} \leqq C_{i}$ pour $1 \leqq i \leqq r$ est $Z_{i}=$ $H_{1} \cdot\left(Z_{i} \cap C_{i}\right)$ pour $r+1 \leqq i \leqq m$. Posons $C=C_{r}$ (si $r$ n'existe pas, on choisit n'importe quel $C \in H_{1}^{\perp}$ ). La condition (iii) de la définition d'un pivot sera satisfaite si on montre que

$$
Z_{i}=\left(Z_{i} \cap H_{1}\right) \cdot\left(Z_{i} \cap C\right) \text { pour tout } 1 \leqq i \leqq m .
$$

Si $i \leqq r, Z_{i} \leqq Z_{r} \leqq C$, donc $Z_{i}=Z_{i} \cap C$. Si $i>r, Z_{i} \geqq H$. Or on a vu ci-dessus qu'on

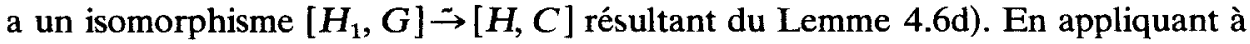
$Z_{i}$ l'aller et retour par cet isomorphisme, on obtient $Z_{i}=H_{1} \cdot\left(Z_{i} \cap C\right)$, comme désiré.

Remarque. Si l'on prend $H=1$ et donc $H_{1}=L_{1}$ dans l'Assertion 2 ci-dessus, 
on retrouve un cas particulier du Théorème 4.7 , à savoir le cas où le sous-groupe normal est minimal, c'est-à-dire simple comme $\mathbb{Z} G$-module. Le Théorème 4.7 en tant que tel ne sera utilisé que dans la Proposition 4.12.

Il est utile d'énoncer le cas particulier $H=1$ dans le Théorème 4.9:

COROLLAIRE 4.10. Soit $1=N_{0} \triangleleft N_{1} \triangleleft \cdots \triangleleft N_{\mathrm{s}}=G$ une suite principale d'un groupe résoluble $G$. Soit $m_{i}$ le nombre de compléments de $N_{i} / N_{i-1}$ dans $G / N_{i-1}$ $(1 \leqq i \leqq s-1)$. Alors $|L(G)|$ a le type d'homotopie d'un bouquet de $m_{1} \cdot m_{2} \cdots m_{s-1}$ sphères de dimension $s-2$.

Remarque. Ce résultat généralise la Proposition 2.6 de [K-T] où seule la caractéristique d'Euler réduite $\tilde{\chi}(L(G))=\mu(1, G)$ était calculée.

COROLLAIRE 4.11. Soit $1=N_{0} \triangleleft N_{1}<\cdots \triangleleft N_{\mathrm{s}}=G$ une suite principale d'un groupe résoluble $G$ et $H$ un sous-groupe de $G$. S'il existe $j$ tel que $N_{j-1}<$ $H \cdot N_{i-1} \cap N_{j}<N_{\mathrm{j}}$, alors $(H, G)$ est contractile. En particulier, $(H, G)$ est contractile si $H$ est compris (proprement) entre deux termes consécutifs d'une suite principale.

Preuve. Si $H \cdot N_{j-1}=H \cdot N_{i}$, alors $H \cdot N_{j-1} \cap N_{j}=N_{i j}$, contrairement à l'hypothèse. Par conséquent, $H \cdot N_{j-1}<H \cdot N_{j}$ et dans les notations du Théorème 4.9, $N_{j-1}=R_{i-1}$ et $N_{j}=L_{i}$ pour un $i$. Si $C$ est un complément de $L_{i} / R_{i-1}$ dans $G / R_{i-1}$, et si $C$ contient $H_{i-1}=H \cdot R_{i-1}=H \cdot N_{j-1}$, alors $N_{j-1}<H \cdot N_{j-1} \cap N_{j} \leqq$ $C \cap N_{j}=C \cap L_{i}=R_{i-1}=N_{j-1}$, ce qui est absurde. Cela signifie que $m_{i}=0$. Ainsi, $|(H, G)|$ est homotope à un bouquet de zéro sphères, donc est contractile.

Remarques. 1) Il est clair que la situation décrite dans ce corollaire ne peut arriver que si $H$ n'est pas normal dans $G$.

2) Si $H \cdot N_{j-1} \cap N_{i}=N_{j-1}$ et si $N_{j} / N_{j-1}=L_{i} / R_{i-1}$ possède des compléments dans $G / N_{j-1}=G / R_{i-1}$, cela ne signifie pas encore que $m_{i}$ est non nul. En effet, il peut arriver que $H \cdot N_{i-1} / N_{j-1}$ ne soit contenu dans aucun complément de $N_{j} / N_{j-1}$, quand bien même il intersecte trivialement $N_{j} / N_{j-1}$.

Pour terminer, on cherche des conditions pour que $L(G)$ soit contractile, toujours sous l'hypothèse que $G$ est résoluble.

PROPOSITION 4.12. Soit $1=M_{0} \triangleleft M_{1} \triangleleft \cdots \triangleleft M_{r}=G$ une suite de sousgroupes normaux de $G$. Pour $1 \leqq i \leqq r$, on suppose que $M_{i} / M_{i-1}$ est abélien (donc $G$ est résoluble), et de plus que $M_{i} / M_{i-1}$ est semi-simple comme $\mathbb{Z}\left[G / M_{i-1}\right]$-module et que $M_{i} / M_{i-1}$ possède un complément dans $G / M_{i-1}$. Alors $L(G)$ n'est pas contractile. 
Preuve. On procède par induction sur $r$. Si $r=1, G$ doit être un $\mathbb{Z}$-module semi-simple (car $G$ agit trivialement), donc un produit de groupes abéliens élémentaires. Par la Proposition 4.5, $L(G)$ n'est pas contractile.

Si $r>1, M_{1}$ est un pivot en vertu du Théorème 4.7. Par hypothèse d'induction, $L\left(G / M_{1}\right) \cong\left(M_{1}, G\right)$ n'est pas contractile et par le Corollaire 4.8 , il suffit de montrer que $\left(1, M_{1}\right)^{H}$ ne l'est pas non plus pour $H \in M_{1}^{\perp}$. Or par le Lemme $4.6 \mathrm{~b}$ ), $\left(1, M_{1}\right)^{H}$ est le treillis des sous- $\mathbb{Z} G$-modules de $M_{1}$. Comme $M_{1}$ est semi-simple par hypothèse, ce treillis est égal au produit des treillis des composantes isotypiques de $M_{1}$. Par la Proposition 2.5, il suffit de montrer que chacun de ces treillis n'est pas contractile. Or, si $M=S \oplus \cdots \oplus S$ ( $k$ facteurs) est un $\mathbb{Z} G$-module semi-simple isotypique et si $\operatorname{End}_{z G}(S)=F$, alors $F$ est un corps fini (commutatif par le théorème de Wedderburn), et par équivalence de Morita, le treillis des sous-modules de $\boldsymbol{M}$ est isomorphe au treillis des sous-espaces d'un $\boldsymbol{F}$-espace vectoriel de dimension $k$. Ce dernier n'est pas contractile par la Proposition 3.6.

Remarquons que la preuve donne en fait une méthode explicite pour calculer le type d'homotopie de $L(G)$ à l'aide de la suite donnée, ceci de manière analogue au Corollaire 4.10. On trouve bien sûr un bouquet de sphères de même dimension, mais leur nombre et la dimension sont décrits par des formules différentes de celles du Corollaire 4.10.

Finalement, voici une proposition qui généralise le Corollaire 2.7 de $[\mathrm{K}-\mathrm{T}]$ :

PROPOSITION 4.13. Soit $G$ un groupe résoluble, $1=N_{0} \triangleleft N_{1} \triangleleft \cdots \triangleleft N_{s}=G$ une suite principale de $G$ et $1=M_{0} \triangleleft M_{1} \triangleleft \cdots \triangleleft M_{r}=G$ une suite caractéristique maximale de $G$. Les conditions suivantes sont équivalentes:

(i) $L(G)$ est contractile.

(ii) $\tilde{\chi}(L(G))=\chi(L(G))-1=0$.

(iii) Il existe $1 \leqq i \leqq s-1$ tel que $N_{i} / N_{i-1}$ ne possède pas de complément dans $G / N_{i-1}$.

(iv) $\Pi$ existe $1 \leqq i \leqq r-1$ tel que $M_{i} / M_{i-1}$ ne possède pas de complément dans $G / M_{i-1}$.

(v) Il existe un sous-groupe caractéristique de $G$ sans complément dans $G$.

(vi) $I$ existe un sous-groupe normal de $G$ sans complément dans $G$.

(vii) $I$ existe un sous-groupe de $G$ sans complément dans $G$ (au sens des treillis).

Preuve. Il est clair que (v) $\Rightarrow($ vi $) \Rightarrow$ (vii) et (vii) $\Rightarrow$ (i) par la Proposition 1.8 . De plus, $|L(G)|$ est homotope à un bouquet de $\tilde{\chi}(L(G))$ sphères, ce qui montre l'équivalence de (i) et (ii). Par le Corollaire 4.10, (i) est équivalent à (iii). Par la 
Proposition 4.12, (i) implique (iv). En effet, $M_{i} / M_{i-1}$ est un sous-groupe caractéristique minimal de $G / M_{i-1}$, donc semi-simple comme $\mathbb{Z}\left[G / M_{i-1}\right]$-module car le radical de $M_{i} / M_{i-1}$ est un sous-groupe caractéristique. Finalement, (iv) implique (v) car si $M_{i} / M_{i-1}$ n'a pas de complément dans $G / M_{i-1}$, alors $M_{i}$ n'en a pas dans $G$.

Remarques. 1) $|L(G)|$ est homotope à un bouquet de sphères de même dimension si $G$ est résoluble. La réciproque n'est en général pas vraie, car $\left|L\left(A_{5}\right)\right|$ a le type d'homotopie d'un bouquet de 60 cercles. Par ailleurs, si $G=P S L_{2}\left(\mathbb{F}_{7}\right)$, le groupe simple d'ordre 168 , on a: $H_{1}(|L(G)| ; \mathbb{Z}) \neq 0$ et $H_{2}(|L(G)| ; \mathbb{Z}) \neq 0$ et de plus $\tilde{\chi}(L(G))=0$. Donc, non seulement $|L(G)|$ n'est pas un bouquet de sphères équidimensionnelles, mais l'équivalence de (i) et (ii) dans la Proposition 4.13 n'a plus lieu pour $G$ non résoluble.

2) Même si, pour $G$ résoluble, tous les intervalles $(H, K)$ ont l'homologie d'un bouquet de sphères équidimensionnelles, $\bar{L}(G)$ n'est pas nécessairement CohenMacaulay. En fait, $\bar{L}(G)$ est Cohen-Macaulay si et seulement si $G$ est hyperrésoluble [ $B_{1}$, p. 167]. Rappelons qu'un treillis est Cohen-Macaulay si pour tout intervalle $(a, b)$, toute chaîne maximale dans $(a, b)$ a la même longueur $d$, et si $(a, b)$ a l'homologie d'un bouquet de sphères de dimension $d-2$.

3) Dans une autre direction, mentionnons le travail de Suzuki [S] sur le treillis $\bar{L}(G)$ des sous-groupes d'un groupe, étudié du point de vue combinatoire. On y trouve par exemple des conditions pour que $\bar{L}(G)$ soit modulaire, semimodulaire, etc.

\section{REFERENCES}

[A] AIGNER, M., Combinatorial theory. Springer Verlag, Berlin (1979).

$\left[\mathrm{B}_{1}\right]$ BJörner, A., Shellable and Cohen-Macaulay partially ordered sets. Trans. Amer. Math. Soc. 260 (1980), 159-183.

$\left[\mathrm{B}_{2}\right]$ BJörner, A., Homotopy type of posets and lattice complementation. J. Comb. Theory A 30 (1981), 90-100.

[F] Folkman, J., The homology group of a lattice. J. of Math. and Mech. 15 (1966), 631-636.

[H] HuPperT, B., Endliche Gruppen I. Springer Verlag, Berlin (1967).

[K-T] Kratzer, C. und Thévenaz, J., Fonction de Möbius et anneau de Burnside. Comment. Math. Helv., 59 (1984), 425-438.

[L] Luszrig, G., The discrete series of $G L_{n}$ over a finite field. Ann. of Math. Studies 81 (1974). Princeton Univ. Press.

[Q] QUILLEN, D., Homotopy properties of the poset of nontrivial p-subgroups of a group. Adv. in Math. 28 (1978), 101-128.

[R] RotA, G.-C., On the foundations of combinatorial theory: I. Theory of Möbius functions. Z. für Wahrscheinlichkeitstheorie und Verw. Gebiete 2 (1964), 340-368. 
[S] Suzuxı, M., Structure of a group and the structure of its lattice subgroups. Ergebn. der Math. 10, Springer Verlag, Berlin (1956).

Université de Lausanne

Institut de Mathématiques

CH-1015 Lausanne

Reçu le 26 Janvier, 1984 